\title{
Numerical and Experimental Study of the Flow Field Structure Evolution in the Circular Recess of Oil Cavity
}

\author{
Feng Shen, Conglian Chen, and Zhaomiao Liu \\ College of Mechanical Engineering and Applied Electronics Technology, Beijing University of Technology, Beijing 100124, China \\ Correspondence should be addressed to Zhaomiao Liu; lzm@bjut.edu.cn
}

Received 22 October 2013; Accepted 10 June 2014; Published 3 July 2014

Academic Editor: Benchawan Wiwatanapataphee

Copyright ( 2014 Feng Shen et al. This is an open access article distributed under the Creative Commons Attribution License, which permits unrestricted use, distribution, and reproduction in any medium, provided the original work is properly cited.

\begin{abstract}
The laminar radial flow in the oil cavity of heavy-duty computer numerical control (CNC) machines is very complicated and has not been fully explored. Navier-Stokes equations have been applied through the whole flow region using finite volume approach to explore this complicated flow phenomenon, including the influences of the clearance height $(h)$, inlet nozzle Reynolds number (Re), and geometrical aspect ratio $(e)$ on flow behaviors. A fluid dynamic experiment has been conducted to study the flow structure by using particle image velocimetry (PIV). Numerical simulation results have been compared with the experimental results, finding a good agreement with the studied cases. The results suggest that there are complex vortices in the oil cavity. Flow field structure of the oil cavity largely depends on $h, \mathrm{Re}$, and $e$. Re and $e$ have a great influence on the size and amount of vortices, and $h$ has slight effects on the size of the vortices. The lengths of primary, secondary, and tertiary isolated vortices have a linear relationship with $h$. The lengths of the primary and secondary isolated vortices increase linearly with ascending $e$ as $e$ is small. But when Re and $e$ are large enough, the size of the three vortices decreases.
\end{abstract}

\section{Introduction}

In recent years, with the rapid development of aerospace, shipbuilding, engineering machinery, and weapons manufacturing industry, there is a higher requirement for $\mathrm{CNC}$ machine tool performance in machining precision or ultrahigh-precision parts. Overloading, high-speed, precision, stability, and automation become the development direction of high-performance CNC machine tools. As the loadbearing platform of high-performance machine, hydrostatic turntable system has an advantage of small frictional resistance, long life, good seismic performance, high accuracy, and good adaptability and is widely used in ultra-precision machine tool and instrument industry. Using oil film as working medium, hydrostatic turntable forms oil pads by using an external pump to inject fluid between the oil pad recesses and the rotary table. In these recesses, the pressure and shear driven flow phenomenon interact in a complicated manner. Therefore, the structure of the flow field in the oil cavity will affect the bearing capacity, stability of the oil film, and the performance of hydrostatic turntable system.
The changes of inlet nozzle pipe Reynolds number Re and geometrical aspect ratio $e$ induce the flow evolution in the entrance section flow field to be extremely complex. It brings about great interest in both the application and research of uncertainty in the related fields. There is great theoretical and application value in revealing how $\operatorname{Re}$ and $e$ influence flow structure characteristics in the oil cavity. As Re and $e$ change, the most significant feature in the entrance section of flow is that different amounts and forms of vortices structure arise. Generally, an annular separation bubble around the inlet corner will lead to the circumferential deviation in the velocity distribution in the downstream region. So there is practical significance to elucidate the size of the separation bubble and the downstream flow structure. Presently, the studies about flow focus mainly on parallel discs and pay much attention to the vortices structure behavior. Sahoo and Sharif examined the details on the size and locations of these vortices affected by the jet Reynolds and Richardson numbers for a laminar confined slot jet [1]. Goldstein and DiMilla used computational fluid dynamics (CFD) to study flow in the RFC at $e=0.2$ at several volumetric flow rates $\left(\mathrm{Re}_{\mathrm{Di}}=1000\right.$, 
1500, and 2000) [2,3]. Van Santen et al. (2000) indicated that the onset of thermal instability became earlier at increasing buoyancy-to-inertia ratio $[4,5]$. Jensen and Friis computed the wall shear stress distribution in a flow going from turbulent to laminar flow conditions between the plates by CFD $(e=0.125)$ [6]. Moller investigated separation bubbles around the inlet corner and radial pressure distributions downstream [7]. Kawaguchi experimentally examined the turbulence about entrance pressure loss and the variation in velocity distributions from the area near the inlet corner to the downstream region [8]. Hsieh et al. observed the flow patterns in the ideal model for chemical vapor deposition (CVD) reactor by employing smoke-tracer flow visualization technique, and their results identified the existence of tertiary isolated vortex and cascaded vortex embedding in the primary isolated vortex for the first time $[9,10]$. Detry et al. investigated the wall shear stress distribution at any distance from the center of the radial axisymmetrical flow for aspect ratios of $0.125,0.25,0.5$, and 1 with inlet Reynolds numbers varying from 0 to 2000 with numerical method, provided a thorough description of the complex flow pattern encountered close to the inlet section, and identified four recirculation zones in both numerical and experimental investigations [11]. Chatterjee used numerical method to study the evolution of the structure of the radial flow field in the channel region beyond the impingement zone and provided a detailed demarcation of the $\mathrm{Re}-e$ plane based on flow separation behavior $[12,13]$. Nakabayashi et al. studied the annular separation bubble that appears around the inlet corner of a radial flow between two parallel discs using flow visualization techniques and numerical simulations and found $\operatorname{Re}$ and $H / d_{p}$ had a great influence on the separation bubble [14]. Vatistas et al. presented a numerical solution to the radial inflow between two flat discs and found that the pressure and the radial velocity distribution were shown to be functions of solely one nondimensional parameter combining the Reynolds number and the radial distance [15]. More complete information on the flow associated with the impinging jets can be found from critical reviews [16, 17].

Unfortunately, all these studies are restricted to limited range of $\operatorname{Re}$ and $e$ and pay little attention to the flow in the oil cavity model which is different from parallel discs model. The sidewall has an effect on the flow and thus the flow field structure has great difference. Therefore, more attention should be paid to research this flow field structure. In addition, utilizing numerical methodology to study radial flow contributes to tackle difficulties which cannot be taken care of by either theoretical or experimental methods. This is a feasible way to complement the research concerning radial flow and to provide guidance for both engineering application and design related to this flow.

This paper investigates the evolution phases of the flow patterns and influence factors of the vortex length with various $h(0.05 \mathrm{~mm}, 0.1 \mathrm{~mm}, 0.2 \mathrm{~mm}$, and $0.4 \mathrm{~mm}), \operatorname{Re}(0 \sim$ $1610)$, and $e(0 \sim 7)$ using numerical simulation method. A fluid dynamic experiment has been conducted to study the flow structure by using particle image velocimetry (PIV). Numerical simulation results have been compared with the experimental results. The relationship between the length of

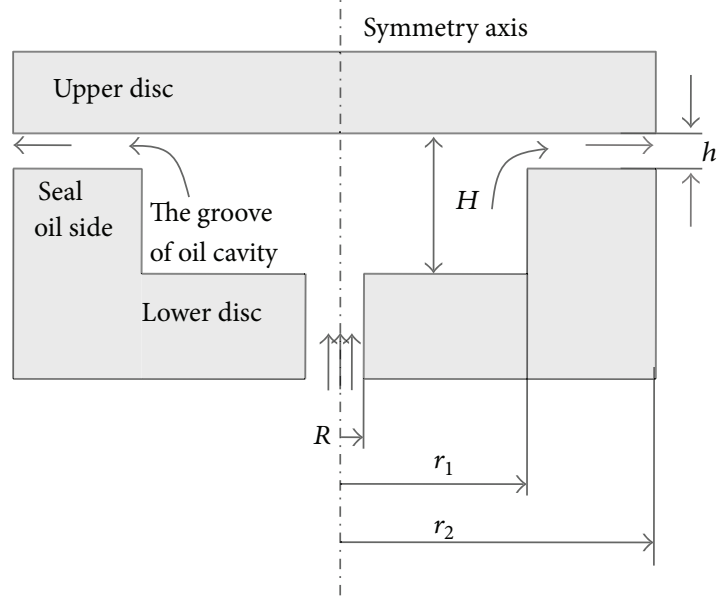

FIGURE 1: 2D geometrical model of the circular oil cavity.

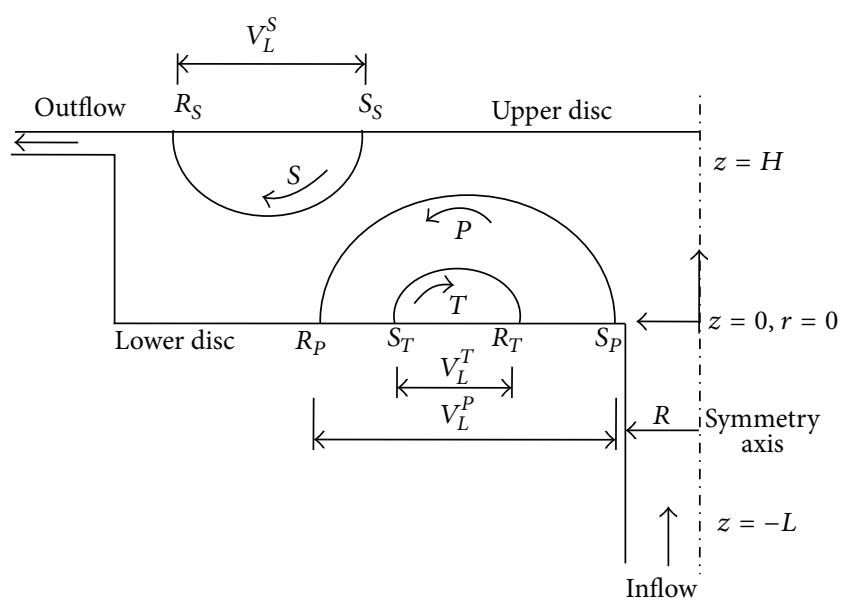

FIGURE 2: 2D geometrical model of flow field structure in oil cavity.

vortices and influence factor is discussed and compared with the results of parallel discs.

\section{Experiment and Numerical Method}

2.1. Oil Cavity Geometrical Model. The shape of the static pressure oil cavity in the high-end CNC machine is generally circular, as shown in Figure 1. The oil cavity is separated from the bearing surface by pressing the working fluid into the groove of the oil cavity. Due to the throttle effect of the seal oil, the oil cavity generates a high pressure to support the external load. The physical description of the basic problems is that when the upper disc is stationary, the flow in the entire oil cavity is three-dimensional axisymmetric flow, and there is no speed around the central axis in the plane of the radial direction. Therefore, the flow can be simplified as a two-dimensional flow in the plane of the radial direction. The geometrical parameters of the shape of the oil cavity are $e=H / R_{i} ; \varepsilon=h / H(0<\varepsilon<1) ; \xi=r_{2} / r_{1} ; r^{*}=r / R$.

Figure 2 shows the 2D geometrical model of flow field structure in the oil cavity, the model consists of two parallel 


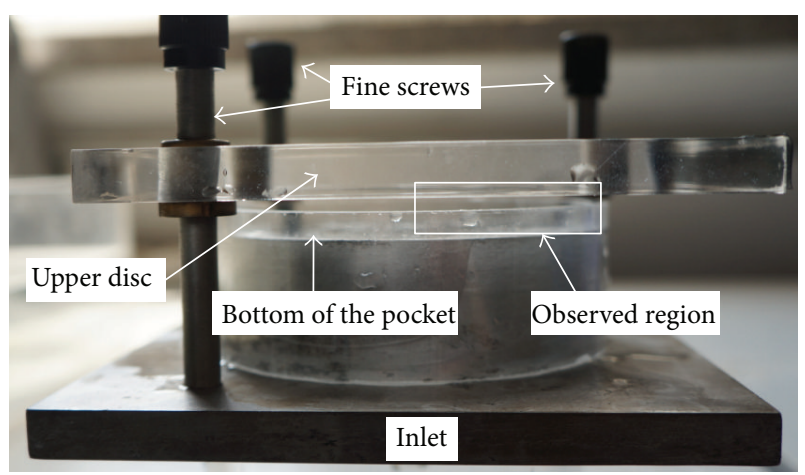

FIgURE 3: Experimental model.

discs and sidewall, and the lower one is connected to a nozzle pipe in its centre. The fluid flows into the nozzle pipe first and then issues from a circular orifice located at the centre of the lower disc into the groove of the oil cavity. The flow field in this geometry can be conveniently divided into three regions [12]: the incoming flow region in the nozzle pipe $(0<r<R$, $-L<z<0$ ), the impinging jet region between the circular orifice and the lower disc $(0<r<R, 0<z<H)$, and the axisymmetric radial flow region in the spacing $(r>R$, $0<z<H)$. A series of flow separation points $(S)$ and reattachment points $(R)$ will appear on both upper and lower discs as the entrance and inertia effect in the entrance section. Corresponding to these flow separation and reattachment points, a series of vortices attached to the upper and lower discs are to be generated.

2.2. PIV System. To verify the accuracy of the numerical simulations, some comparisons have been carried out with the experimental results obtained by particle image velocimetry (PIV) technique. PIV is an optical method of flow visualization used to obtain instantaneous velocity measurements and related properties of fluids [18-20]. Polyamide seeding particles (diameter of the particles is about $20 \mu \mathrm{m}$ and density is $1.03 \sim 1.05 \mathrm{~g} / \mathrm{cm}^{3}$ ) are used as PIV tracers in the flow field. The fluid is seeded with polyamide seeding particles, which, for sufficiently small particles, are assumed to faithfully follow the flow dynamics. For the PIV measurement, double pulsed DANTEC DualPower lasers are used to supply a pulsed laser sheet (thickness of the sheet is about $1.0 \mathrm{~mm}$ ) with the frequency of $7.4 \mathrm{~Hz}$ and power of $20 \mathrm{~mJ} /$ pulse to illuminate the measured flow field. A 2048 by 2048 pixels cross-correlation CCD array camera (Flowsense) is used to capture PIV images. The twin DANTEC DualPower lasers and the CCD camera are controlled by a synchronizer control system. The PIV images captured by the CCD camera are digitized by an image processing board, then transferred to a workstation for image processing, and displayed on a PC monitor. The fluid with entrained particles is illuminated so as to be visible.

The advantage of PIV is that it can record a great quantity of flow information for fluid particles without intrusive flow measurement probes. The method is, to a large degree, nonintrusive and capable of measuring an entire two-dimensional cross section of the flow field simultaneously. The added seeding particles generally cause negligible distortion of the fluid flow. Optical measurement avoids the need of pitot tubes, hotwire anemometers, or other intrusive flow measurement probes.

\subsection{Experimental Setup and Parameter Settings. According to} the $2 \mathrm{D}$ geometrical model of oil cavity shown in Figure 1, we design and process a circular oil cavity experimental model as shown in Figure 3. The upper disc is made of plexiglass and the lower disc which is connected with a $6 \mathrm{~mm}$ diameter central inlet pipe is made of aluminium alloy. The distance between these two discs is set by three adjustable fine screws fixed in the upper disc. The depth of the oil cavity $(H)$ is $12 \mathrm{~mm}$. The oil cavity has an inside radius $\left(r_{1}\right)$ of $45 \mathrm{~mm}$ and the inlet radius of nozzle pipe $(R)$ is $3 \mathrm{~mm}$. The distance between seal oil side and upper $\operatorname{disc}(h)$ is $1 \mathrm{~mm}$ and the width of the seal oil side is $r_{2}-r_{1}=5 \mathrm{~mm}$. The fluid is deionized water and the temperature is $20^{\circ} \mathrm{C}$.

Schematics of the experimental system and test section are, respectively, shown in Figures 3 and 4 . The system consists of the oil cavity model, lasers, synchronization controller, CCD camera, and computer. The flow in the test loop is supplied from a head tank, which is continuously pump-filled from a lower tank. The fluid goes into the lower tank when it flows out from the model. The water level in the head tank is maintained constant by an overflow system in order to eliminate the effect of the pump vibration on the inlet condition of the test tank. The flow rate of the flow loop, which is used to calculate the representative velocity and Reynolds numbers, is measured by a flow meter.

The fluid passes through a surge tank to suppress the pulses from the pump before it is entering the inlet pipe. During the experiment, the polyamide seeding particles have been poured into deionized water. The spacing flow region is illuminated from one side by a laser pulse sheet passing through the center of the upper disc and paralleling to the shooting surface. Images are to be captured by a high-speed digital camera after the laser pulse, whose pulse frequency is set in accordance with shooting frequency. Observation has been carried out only on one half of entire flow region as shown in Figure 3 as for the symmetrical characteristic of axisymmetric radial flow.

As the circular sidewall of the oil cavity has an effect on the light refraction and produces an additional lens effect, the particle images will be distorted, inducing an error source for PIV velocity measurements. To reduce this effect, we design a rectangular sink to place the experimental model. The space of the flow region is designed to be submerged in water so as to avoid any optical disturbance due to the presence of water surface between the camera and laser sheet, as shown in Figure 5. The uncertainty in the PIV velocity measurements was estimated to be less than $3 \%$ of the flow velocity in the recess.

2.4. Numerical Methods. We consider incompressible viscous fluid flow of constant viscosity and simulate the behavior of a sufficiently developed Poiseuille flow flowing into the 


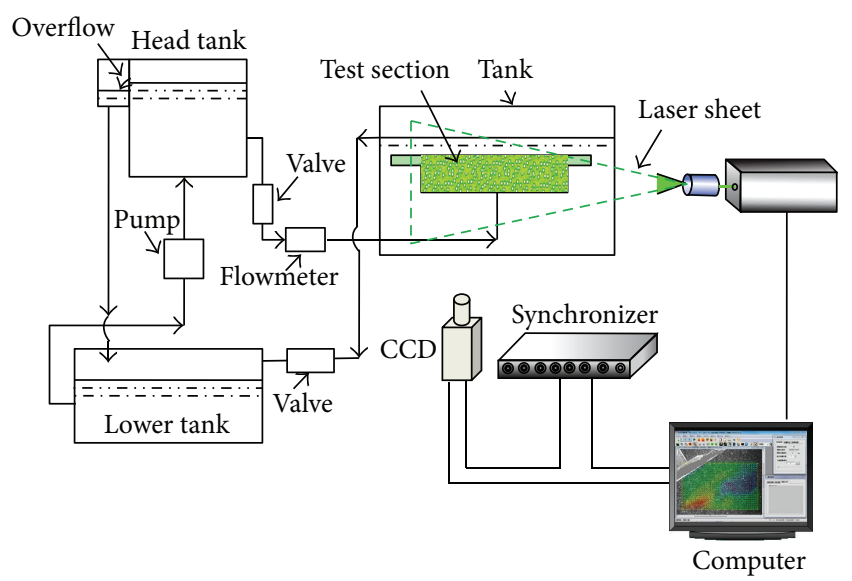

Figure 4: The schematic of the experimental setup.

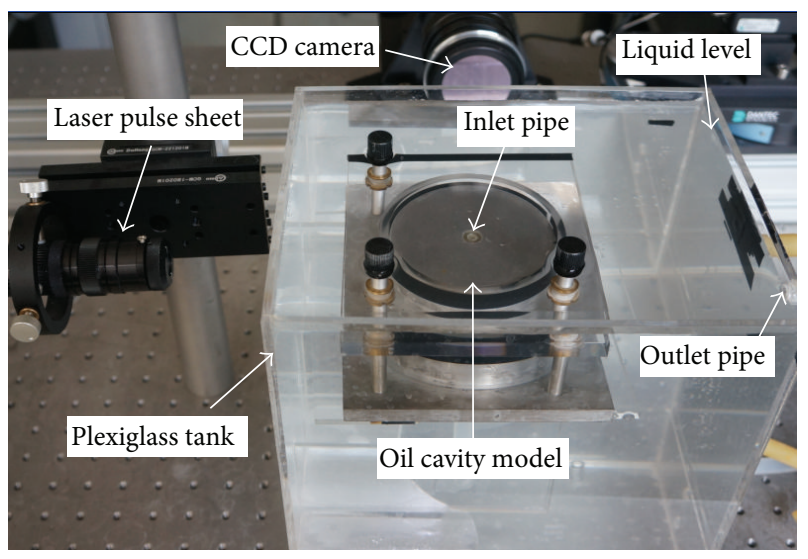

FIgURE 5: Experimental platform.

oil cavity, supposing a two-dimensional axisymmetric flow. The governing equations for the flow are the incompressible Navier-Stokes and continuity equations in axisymmetric cylindrical coordinate systems. Boundary conditions are set in such that there is no velocity along the wall surface, with velocity arranged along the boundary so as to satisfy the equation of continuity in the outflow. For the axial velocity in the supply pipe, the mean velocity is $U$. The inlet velocity is constant.

The flow between the discs is considered as laminar when the disc spacing is narrow and the inlet Reynolds number is low enough $(\operatorname{Re}<2000)$ [11]. The whole flow field is assumed to be steady and isothermal laminar flow according to the range of Re and $e$ selected here. A fully developed speed distribution at the entrance of inlet nozzle pipe is employed as inlet boundary condition, while the outlet boundary condition is a fixed static pressure (an atmosphere) at the outlet of spacing which has no slip at the walls.

The flow domain is limited to an axisymmetrical section of the oil cavity. The numerical simulation model of the oil cavity is shown in Figure 6. In the axial direction the whole inlet $(60 \mathrm{~cm}$ length, $3 \mathrm{~mm}$ radius) is represented to ensure fully developed flow at the inlet turn. Mesh independency

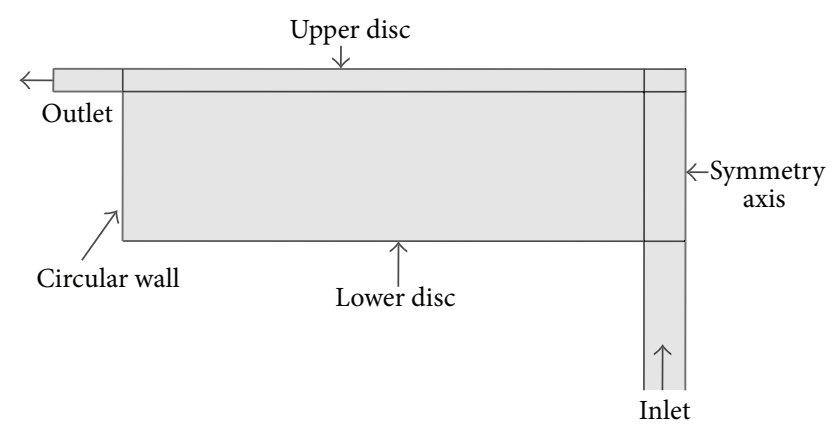

FIGURE 6: Numerical simulation model of the oil cavity.

is investigated. Total number of cells is, respectively, 49571, 126396, 244126, and 304306. Simulations are performed in the 304306 cells mesh on the whole range of Re from 70 tol610 with water as the working fluid, in order to compare the simulation results with the experiments.

\section{Results and Discussion}

The axisymmetric radial flow field in oil cavity is mainly governed by three parameters: the inlet nozzle pipe Reynolds number Re, geometrical aspect ratio, and distance between seal oil side and upper disc $h$. The distance between two parallel discs is modulated by aspect ratio $e$. The lubricant is assumed to be incompressible and isoviscous (i.e., there is negligible variation of viscosity with pressure or temperature). Due to the low inlet flow rate, flow in the recess is assumed to be steady. The continuity and conservative form of Navier-Stokes equations are given in (1) and (2), respectively, as follows:

$$
\begin{gathered}
\nabla \cdot \mathbf{v}=0, \\
\rho(\mathbf{v} \cdot \nabla) \mathbf{v}=\rho \mathbf{f}-\nabla p+\mu \nabla^{2} \mathbf{v},
\end{gathered}
$$

where $\mu=$ dynamic viscosity, $\rho=$ density, $\mathbf{v}=(u, v)^{T}$ velocity vector, $p=$ pressure, $\mathbf{f}=$ external force field, which is assumed to be zero, and $\nabla=(\partial / \partial x) i+(\partial / \partial y) j$.

The local Reynolds number between the discs, the Reynolds number in the inlet pipe, and the wall shear stress are given by (3), (4) and (5), respectively [7]:

$$
\begin{aligned}
& \operatorname{Re}=\frac{\rho \cdot Q}{\pi \cdot \mu \cdot R_{i}}, \\
& \operatorname{Re}_{r}=\frac{2 \cdot \rho \cdot Q}{\pi \cdot \mu \cdot r}, \\
& \tau=\frac{3 \cdot \mu \cdot Q}{\pi \cdot r \cdot H^{2}},
\end{aligned}
$$

where $\rho=$ density, $Q=$ volumetric flow rate, $\mu=$ dynamic viscosity, $r$ = radius of the inlet pipe, $R_{i}=$ radial position from the inlet, and $H=$ distance between two parallel discs.

3.1. Comparisons of the Experimental Results and Numerical Simulations. Prediction of the flow pattern with the coarser 


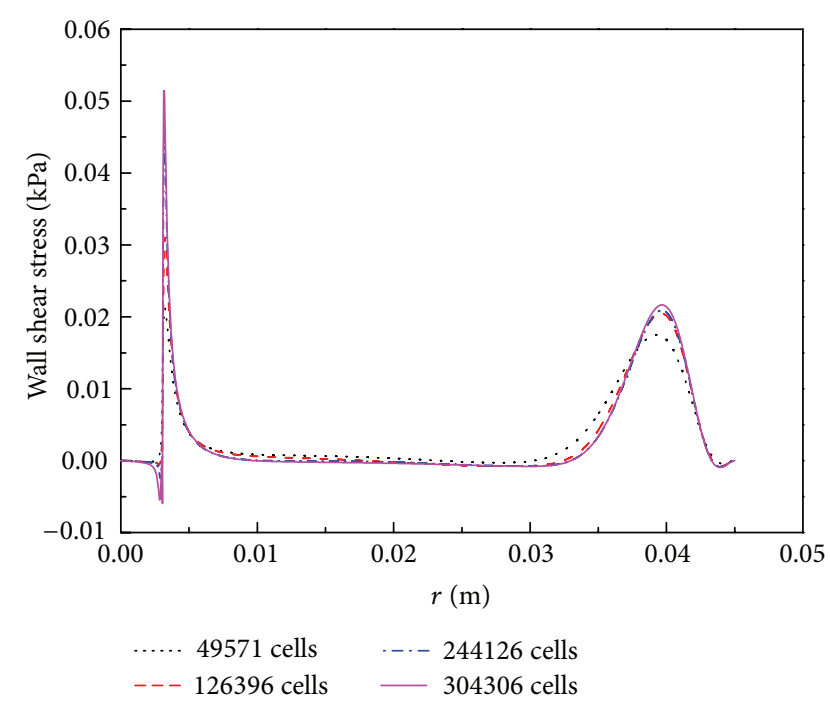

Figure 7: Wall shear stress at line of $0.01 \mathrm{~h}$ from the lower wall of the oil cavity at $\mathrm{Re}=1190$.

mesh (49571 cells) shows the presence of two recirculation zones in the oil cavity at the lower disc as Figure 7 shows. Any further increasing in the number of cells (to 126396, 244126, and 304306) leads to the displacement of the end of tertiary isolated vortex. The refinement of the mesh in the computational domain between 49571 and 304306 cells present for $e=4$ leads thus to an increasing accuracy of the flow structures. Mesh independence of recirculation zone extensions is seen between 244126 and 304306 cells, suggesting that the model give relevant estimation of the wall shear stress and locations of recirculation zones for laminar flows in the inlet pipe. At $\operatorname{Re}=1190$, the difference between the wall shear stress values computed for the 244126 cells mesh and for the 304306 cells mesh is lower than $5 \%$ on most of the flow domain. As this slight difference is observed for a number of cells, mesh independence is considered at this volumetric flow rate.

Three inlet Reynolds numbers are designed to be investigated at aspect ratio $e=4: \operatorname{Re}=210, \mathrm{Re}=630$, and $\mathrm{Re}=1540$. A large amount of flow pattern pictures is to be taken at set intervals for every inlet Reynolds number. Then these pictures are superposed together so the traces of particles in the flow region can be distinctly presented with also the flow pattern. The comparisons of radial flow patterns at three different Reynolds numbers are shown in Figure 8, suggesting that the radial flow patterns expressed through numerical simulation are in accordance with those through PIV particle trace images in aspects of number, shape, and size of vortices structures for all three different Reynolds numbers. Therefore, the numerical simulations here can be considered feasible and practical.

\subsection{Characteristics of Flow Field Structures with Various} Factors. Figure 9 shows the stream function contour plots at various values of $h$ as $\operatorname{Re}=1190$ and $e=2$. As $h$ rises from $0.05 \mathrm{~mm}$ to $0.4 \mathrm{~mm}$, the structure of the flow field is essentially unchanged. There are three vortices anchoring along the surface, $z=0$ and $z=H$, named the primary, secondary, and tertiary vortices. The size of the primary vortices remains stable, but the sizes of the secondary and tertiary vortices increase with $h$, as shown in Figures 12-14. This is due to the restriction of the sidewall to the vortices becoming weak as $h$ increases.

Contour plots of the stream function are depicted in Figure 10, which shows the occurrence of multiple vortices at different values of $\operatorname{Re}$ and a fixed value of $e=3$ and $h=0.1 \mathrm{~mm}$. It is clear that, as Re increases from the creeping flow limit and inertia becomes significant, successive flow separations occur and lead to the appearance of the primary, secondary, and tertiary vortices, the primary and tertiary being anchored along the surface $z=0$ while the secondary is anchored at $z=H$. The primary and secondary vortices move to the sidewall as Re increases. Therefore, the secondary vortex disappears at last at the effect of the pressing action of primary vortex and sidewall, just as Figure 10(c) shows.

Based on the observations from Figure 11, for a given Re, at sufficiently small values of the aspect ratio $e$, presumably due to strong axial vortex diffusion, vortex abstraction at the surface $z=0$, which is necessary to cause separation, is suppressed. On the other hand, at large values of $e$ axial vortex diffusion is weak but is the average circulation within the primary vortex, and conditions once again are not conducive to flow separation within the primary vortex. However, at critical values of the aspect ratio, vortex abstraction is sufficiently strong and leads to separation resulting in the formation of the tertiary vortex which is anchored along the surface $z=0$ and is wholly enclosed within the counter clockwise primary vortex. This tertiary vortex rotates in a clockwise direction, as drawn in Figure 2. In summary, from these results we conclude that even at high Reynolds numbers the fine structure of the steady laminar developing flow field continues to display complex transitions and depends on the aspect ratio in an essential manner.

\subsection{Effects of the Clearance Height $(h)$ on Isolated Vortices.} Because of the contour profile irregularity of all the vortices, the dimensionless distance between the flow separation and the reattachment points of each vortex is selected as its characteristic length [13]. Wall shear stress is chosen to define the separation and the reattachment points. When the sign of the wall shear stress changes, separation or reattachment points appear. Figure 12 plots the dependence of the dimensionless length $\left(V_{L}^{P}\right)$ of primary isolated vortex on $h$ for various $\operatorname{Re}$ as $e=2$. It is discovered that $h$ has small influence on primary isolated vortex length $V_{L}^{P}$. There is a linear relationship between $h$ and $V_{L}^{P}$ and $V_{L}^{P}=\alpha h+\beta$. However, dimensionless tertiary isolated vortex length $\left(V_{L}^{T}\right)$ increases gradually with raising $h$, as shown in Figure 14, and there is a remarkable linear relationship between $V_{L}^{T}$, $h$, and $V_{L}^{T}=\alpha^{*} h+\beta^{*}$. From Figure 13 it is apparent that for $\mathrm{Re}<1190$ the dimensionless secondary isolated vortex length $\left(V_{L}^{S}\right)$ scales linearly with $h$ and $V_{L}^{S}=\alpha h+\beta$. When $\operatorname{Re}>1400, V_{L}^{S}$ decreases gradually with raising $h$. The slope 

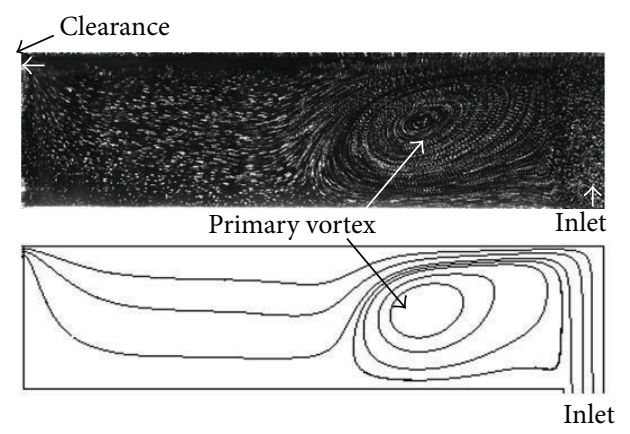

(a)
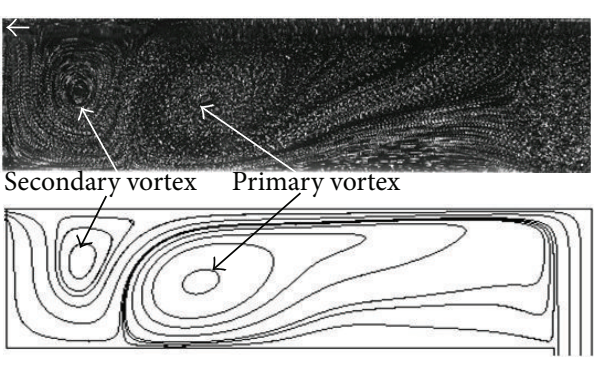

(b)
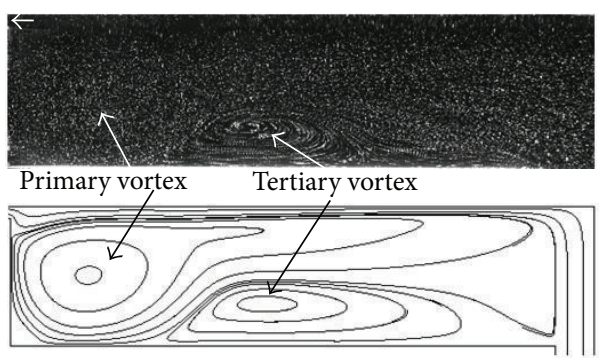

(c)

FIGURE 8: Comparisons of PIV particle images (upper) and numerical simulated streamlines (lower) for radial flow pattern of $e=4, h=1 \mathrm{~mm}$ in oil cavity: (a) $\operatorname{Re}=210$, (b) $\operatorname{Re}=630$, and (c) $\operatorname{Re}=1540$.

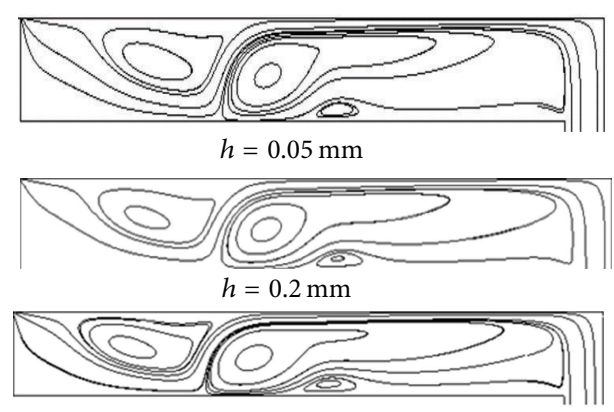

$h=0.4 \mathrm{~mm}$

FIGURE 9: Stream function contour plots at various values of $h$ as $\operatorname{Re}=1190, e=2$.

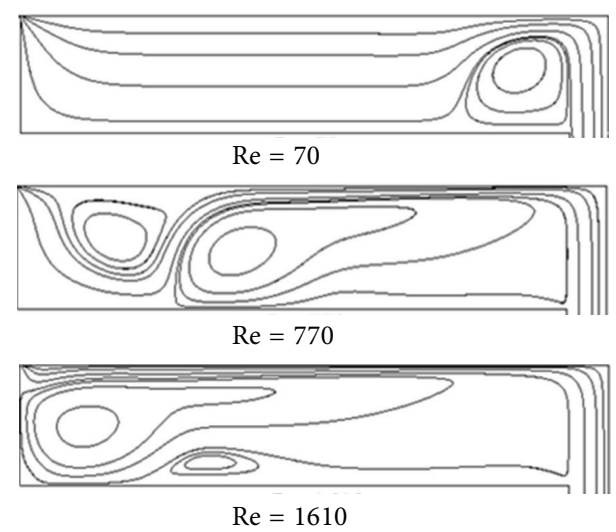

FIGURE 10: Stream function contour plots at various values of Re as $e=3, h=0.1 \mathrm{~mm}$. value $\alpha$ and intercept value $\beta$ at various Re are listed in Table 1 . Furthermore, the higher $R^{2}$ is, the better fitting accuracy will be.

Figure 15 displays the dependence of the dimensionless length $\left(V_{L}^{P}\right)$ of primary isolated vortex on Re for various $h$ as $e=2$. It can be observed that $V_{L}^{P}$ increases gradually with raising Re for various $h$, and there is a power function relationship between $V_{L}^{P}$ and $\operatorname{Re}(\operatorname{Re}>70)$, but no longer a linear relationship. The dependence of the dimensionless length $\left(V_{L}^{S}\right)$ of secondary isolated vortex on Re for various $h$ is shown in Figure 16. When Re $<1190, V_{L}^{S}$ increases along with ascending $h$ as well, yet no specific elementary function relationship can be figured out to demonstrate how $V_{L}^{S}$ is influenced by Re. Due to the mutual restraint between primary and secondary isolated vortices, the nonlinear relationship between $V_{L}^{P}$ and $V_{L}^{S}$ increases along with Re. As these two vortices are large enough, the outward radial growth of primary isolated vortex is restrained by the inward radial growth of secondary isolated vortex. When Re > 1200, as the secondary isolated vortex is close to sidewall and restrained by the sidewall, the dimensionless length $\left(V_{L}^{S}\right)$ of secondary isolated vortex decreases. Figure 17 displays the dependence of the dimensionless length $\left(V_{L}^{T}\right)$ of tertiary isolated vortex on $\operatorname{Re}$ for various $h$ as $e=2$. It suggests that the dimensionless length of tertiary isolated vortex $V_{L}^{T}$ and Re have a relationship of approximate linear growth as $\mathrm{Re}$ between 910 and 1610 .

3.4. Effects of the Geometrical Aspect Ratio (e) Isolated Vortices. Figure 18 shows the dependence of the dimensionless length 
TABLE 1: Slope value $\alpha$ and intercept value $\beta$ of expression for various Re.

\begin{tabular}{cccccccccccccccc}
\hline & \multicolumn{3}{c}{$\mathrm{Re}=280$} & \multicolumn{3}{c}{$\mathrm{Re}=490$} & \multicolumn{3}{c}{$\mathrm{Re}=910$} & \multicolumn{3}{c}{$\operatorname{Re}=1190$} & \multicolumn{4}{c}{$\operatorname{Re}=1610$} \\
& $V_{L}^{P}$ & $V_{L}^{S}$ & $V_{L}^{T}$ & $V_{L}^{P}$ & $V_{L}^{S}$ & $V_{L}^{T}$ & $V_{L}^{P}$ & $V_{L}^{S}$ & $V_{L}^{T}$ & $V_{L}^{P}$ & $V_{L}^{S}$ & $V_{L}^{T}$ & $V_{L}^{P}$ & $V_{L}^{S}$ & $V_{L}^{T}$ \\
\hline$\alpha$ & 0.627 & 1.005 & $/ /$ & 0.800 & 0.765 & $/ /$ & 0.897 & 0.623 & 0.616 & 0.821 & 0.403 & 1.104 & 0.432 & -0.139 & 2.044 \\
$\beta$ & 4.698 & 1.567 & $/ /$ & 6.139 & 2.732 & $/ /$ & 7.912 & 3.870 & 0.670 & 8.786 & 4.194 & 1.820 & 9.968 & 4.079 & 2.989 \\
$R^{2}$ & 0.993 & 0.999 & $/ /$ & 0.999 & 0.999 & $/ /$ & 0.997 & 0.996 & 0.999 & 0.998 & 0.996 & 0.998 & 0.995 & 0.993 & 0.999 \\
\hline
\end{tabular}
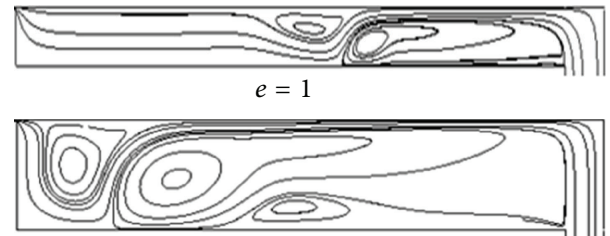

$e=3$

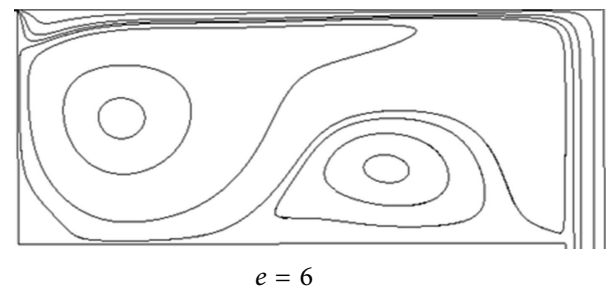

FIGURE 11: Stream function contour plots at various values of aspect ratio $e$ as $\operatorname{Re}=1190, h=0.1 \mathrm{~mm}$.

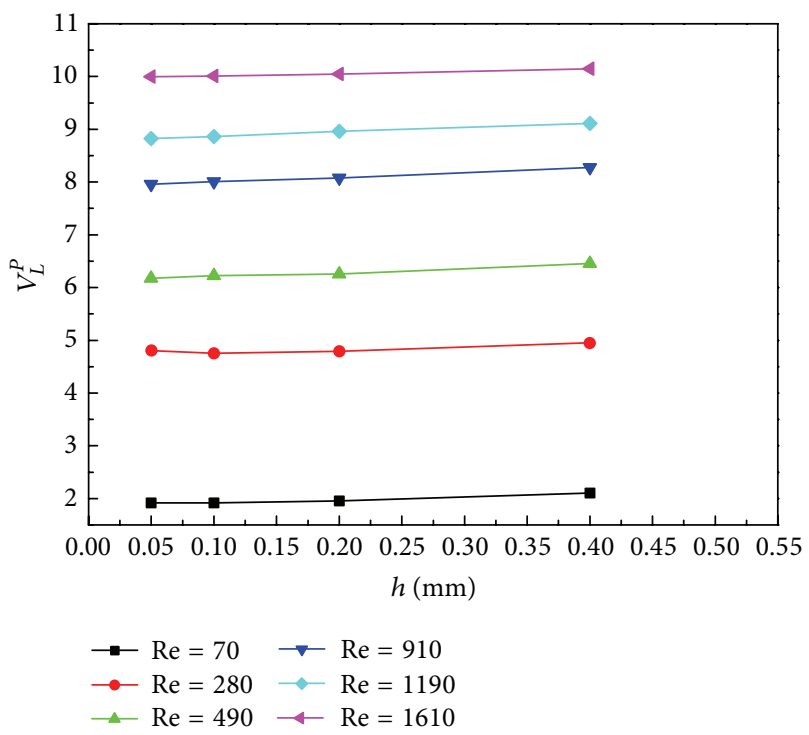

FIGURE 12: Dimensionless primary vortex length $V_{L}^{P}$ as a function of $h$ for various $\operatorname{Re}$ as $e=2$.

$\left(V_{L}^{P}\right)$ of primary isolated vortex on $e$ for various $\operatorname{Re}$ as $h=$ $0.1 \mathrm{~mm}$. It is apparent that for small Reynolds number $(140<$ $\operatorname{Re}<280)$ the primary isolated vortex length $\left(V_{L}^{P}\right)$ scales linearly with the aspect ratio $e$ for $1<e<7$. When $\operatorname{Re}>560$, the primary isolated vortex length $V_{L}^{P}$ increases linearly at small $e$ and decreases at large $e$. As Re increases, the primary isolated vortex length $V_{L}^{P}$ diminishes largely and this decreasing phenomenon appears at smaller $e$. The confine of

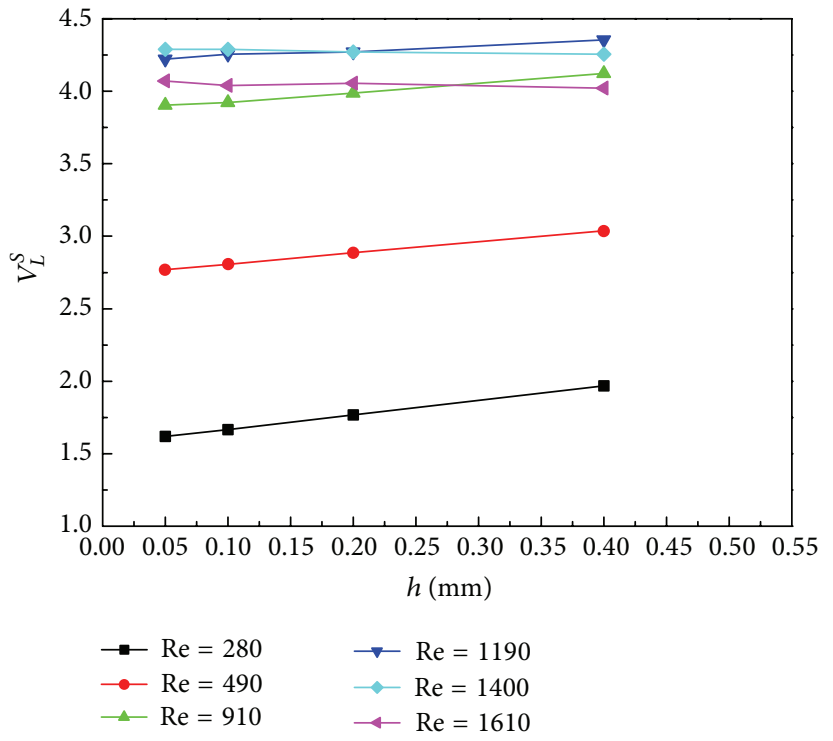

FIGURE 13: Dimensionless secondary vortex length $V_{L}^{S}$ as a function of $h$ for various $\operatorname{Re}$ as $e=2$.

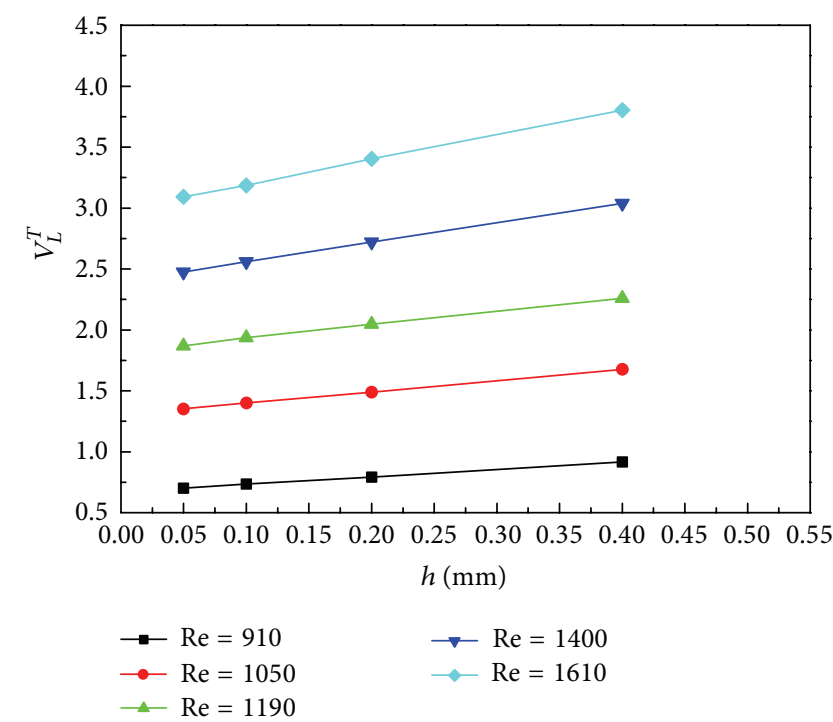

FIGURE 14: Dimensionless tertiary vortex length $V_{L}^{T}$ as a function of $h$ for various $\operatorname{Re}$ as $e=2$.

the sidewall is the reason for this phenomenon. When Re is small, primary isolated vortex is small and far away from the sidewall, and the flow accords with axisymmetric radial flow in two discs. Therefore, $V_{L}^{P}$ increases linearly with $e$. When Re increases, primary isolated vortex moves to the sidewall 


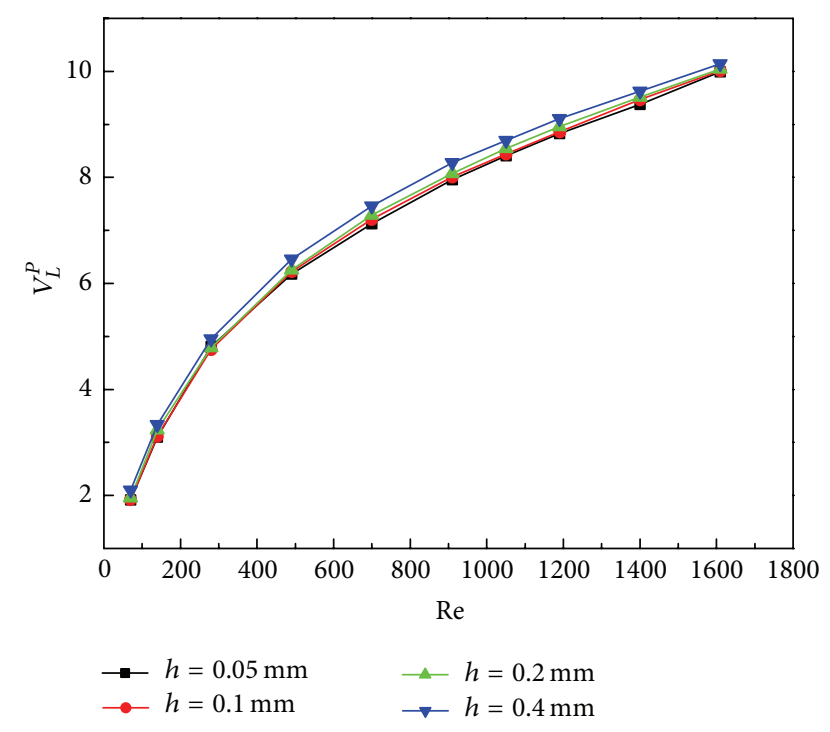

FIGURE 15: Dimensionless primary vortex length $V_{L}^{P}$ as a function of Re for various $h$ as $e=2$.

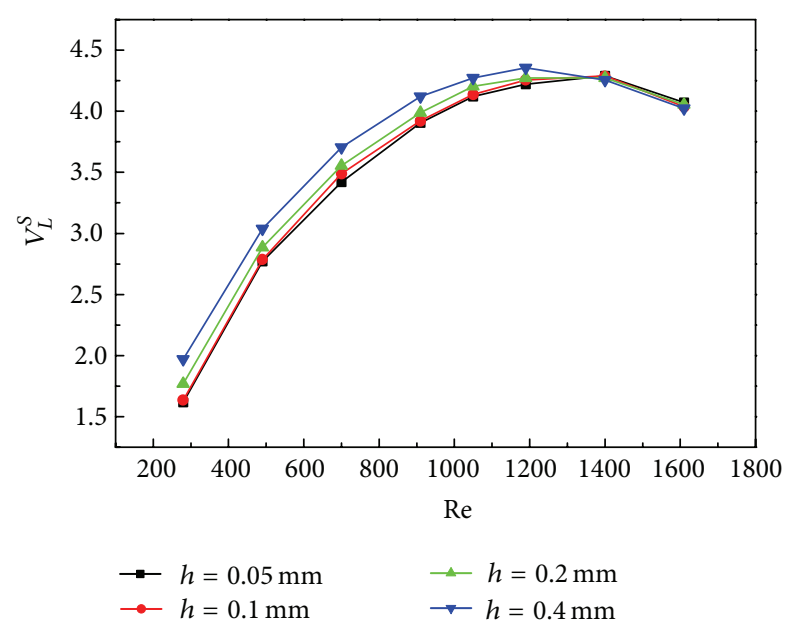

FIGURE 16: Dimensionless secondary vortex length $V_{L}^{S}$ as a function of Re for various $h$ as $e=2$.

along the radial direction. The primary isolated vortex gets close to the sidewall as Re and $e$ are large enough. For this reason, the development of the primary isolated vortex is confined and $V_{L}^{P}$ decreases. The secondary isolated vortex is also affected by the sidewall, as Figure 19 shows. When $\operatorname{Re}=350$, the secondary isolated vortex length $V_{L}^{S}$ increases gradually with raising $e$. However, when $420<\mathrm{Re}<700$, the secondary isolated vortex length $\left(V_{L}^{S}\right)$ increases at smalle and decreases at large $e$. This is due to the compression effect of primary isolated vortex and the sidewall. Figure 20 shows the dependence of the dimensionless length $\left(V_{L}^{T}\right)$ of tertiary isolated vortex on $e$ for various Re. With ascending $e, V_{L}^{T}$ firstly rises to its maximum value and then declines gradually. From the development tendency of the curves in Figure 20, it

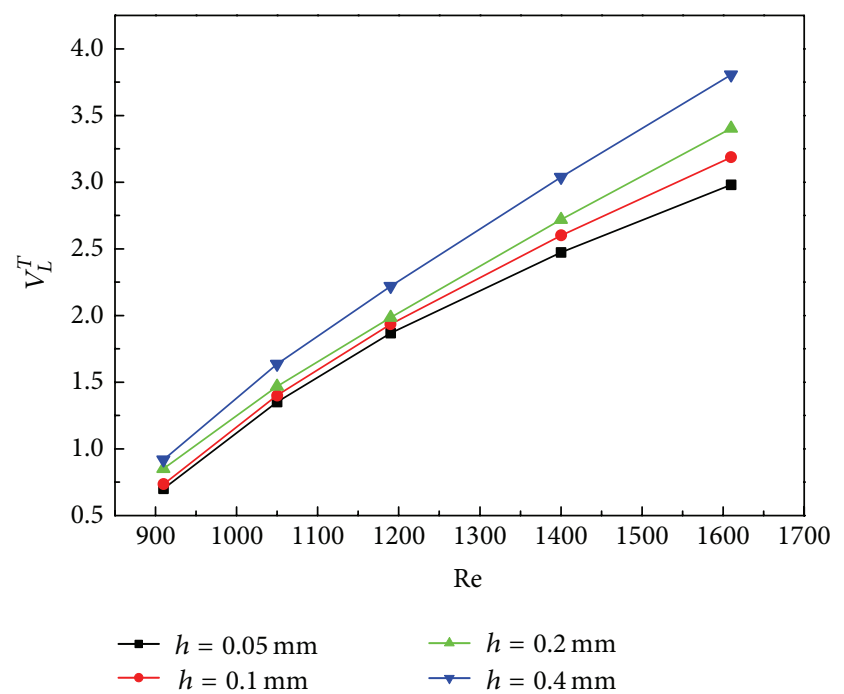

FIGURE 17: Dimensionless tertiary vortex length $V_{L}^{T}$ as a function of Re for various $h$ as $e=2$.

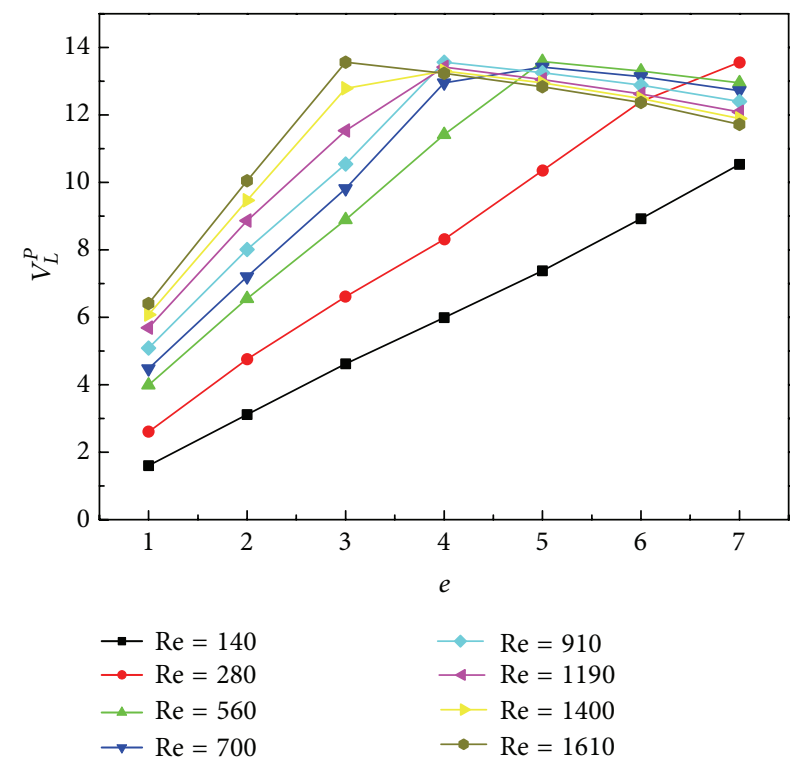

FIgURE 18: Dimensionless primary vortex length $V_{L}^{P}$ as a function of $e$ for various $\operatorname{Re}$ as $h=0.1 \mathrm{~mm}$.

can be concluded that the tertiary isolated vortex only exists in the axisymmetric radial flow field region at a specific finite range of $e$ corresponding to different Re.

Figures 21, 23, and 24 show the dependence of the vortex lengths of the primary, secondary, and tertiary isolated vortices as a function of Re for various $e$ as $h=0.1 \mathrm{~mm}$. All three vortices increase in size at small Reynolds number. For the primary isolated vortex, $V_{L}^{P}$ increases gradually with raising Re as $e \leq 3$, while as $e>3, V_{L}^{P}$ increases at small $\mathrm{Re}$ and decreases at large Re. The critical Re of this change has a relationship with $e$. With the increasing of $e$, the critical Reynolds number Re decreases, as Figure 22 shows. The secondary isolated vortex appears at $e \leq 4$. When $e=1$ and 2 , 


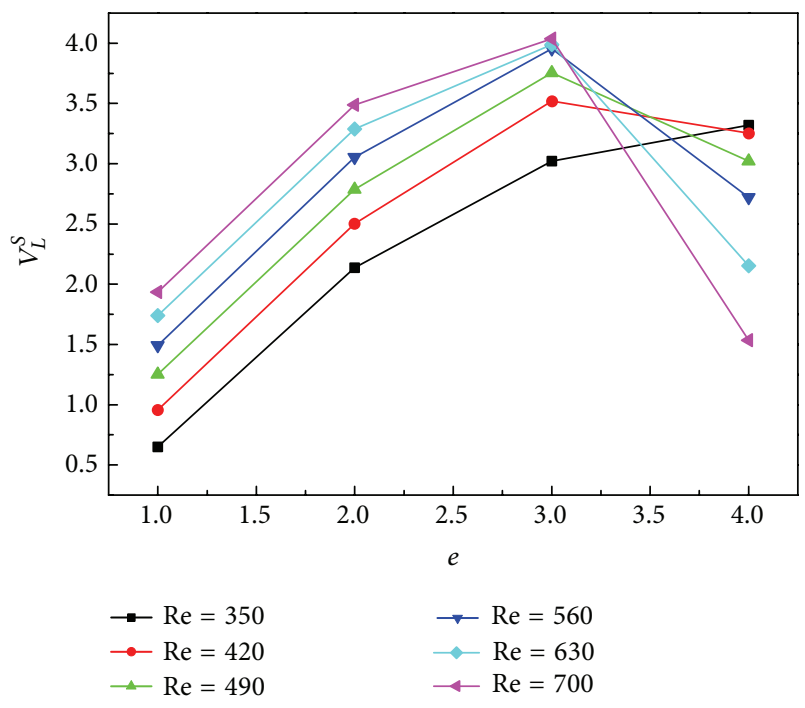

FIGURE 19: Dimensionless secondary vortex length $V_{L}^{S}$ as a function of $e$ for various $\operatorname{Re}$ as $h=0.1 \mathrm{~mm}$.

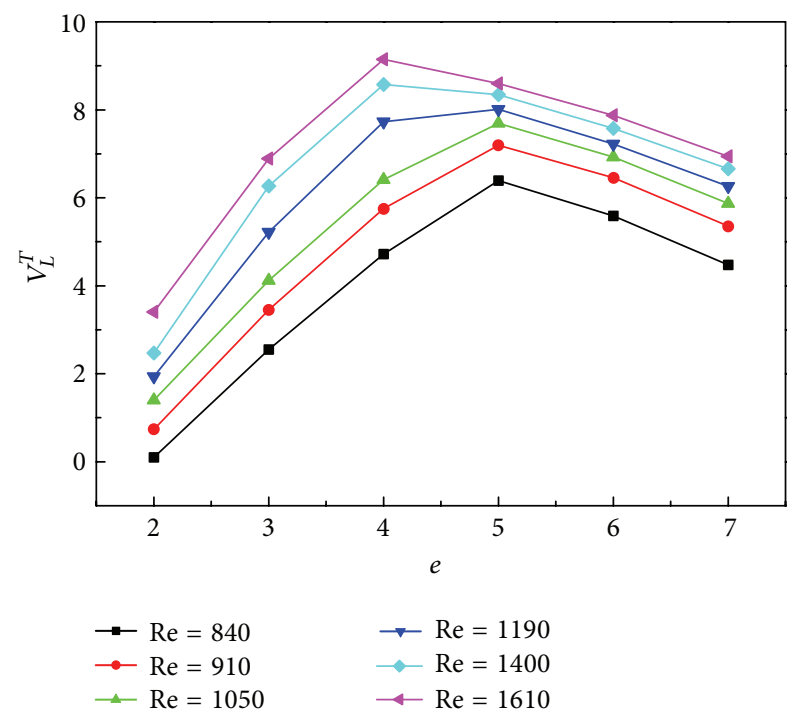

FIGURE 20: Dimensionless tertiary vortex length $V_{L}^{T}$ as a function of $e$ for various $\operatorname{Re}$ as $h=0.1 \mathrm{~mm}$.

the secondary isolated vortex length $V_{L}^{S}$ increases nonlinearly as Re rises. However, as $e=3$ and 4 , due to the pressing action of sidewall and the primary vortex, the secondary isolated vortex length decreases and vanishes at last. The tertiary isolated vortex length $V_{L}^{T}$ increases rapidly with Reynolds number for various $e$. When $e \leq 3$, tertiary isolated vortex length $\left(V_{L}^{T}\right)$ rises as $e$ increases. However, when $e \geq 4$, the result is reversed. The reason of this phenomenon is that the effect of fluid inertia decreases and makes the flow field more complex as $e$ increases.

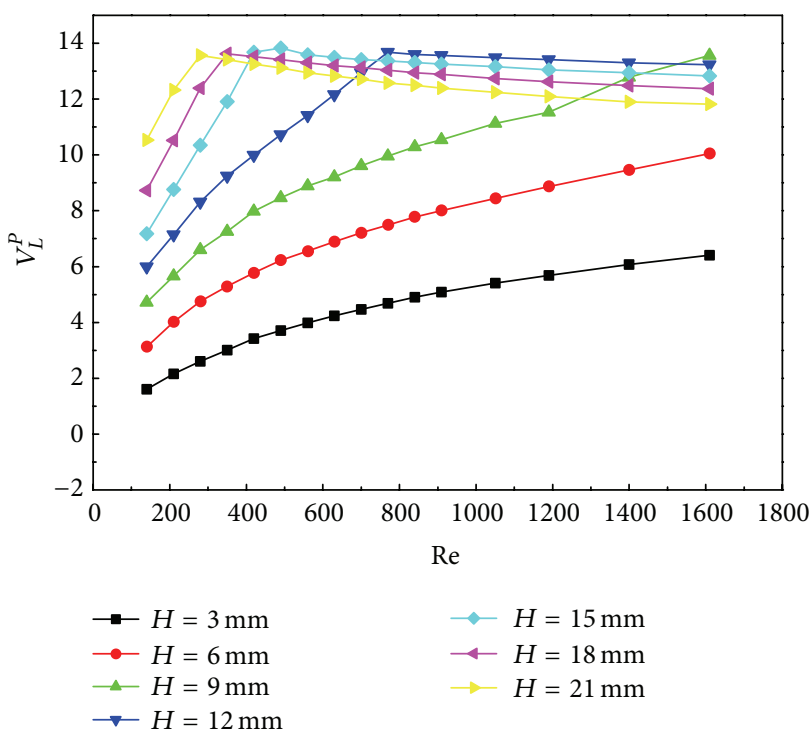

FIgURE 21: Dimensionless primary vortex length $V_{L}^{P}$ as a function of Re for various $e$ as $h=0.1 \mathrm{~mm}$.

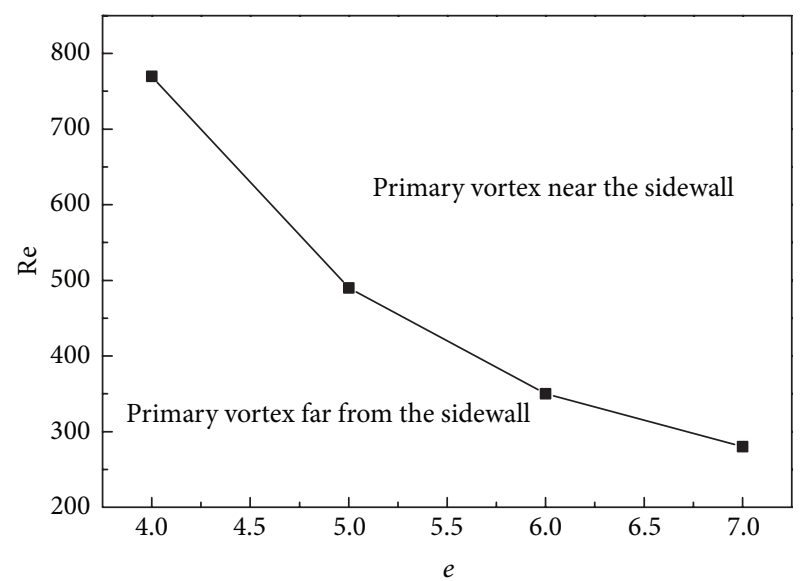

FIgURE 22: Demarcation of the $\mathrm{Re}-e$ plane corresponding to sidewall effecting primary vortex as $h=0.1 \mathrm{~mm}$.

\section{Conclusions}

It is necessary to study a single circular recess by solving the Navier-Stokes equations utilizing a finite volume approach and PIV experiments due to the complexity of the radial flow processes and realistic operating conditions of the hydrostatic rotary table. A CFD model is developed in the present work in order to establish the liaison between experimental measurements performed in the oil cavity and numerical simulation results. Mesh independence is tested. Moreover, the evolution of the flow pattern with the clearance height, the volumetric flow rate and the geometrical aspect ratio is explored. Three isolated vortices are identified and the size of the vortices is measured. Numerical simulation results have been compared with the experimental results and have a good match. Flow field structure of the oil cavity largely depends on $h, \operatorname{Re}$, and $e$. Re and $e$ have a great influence 


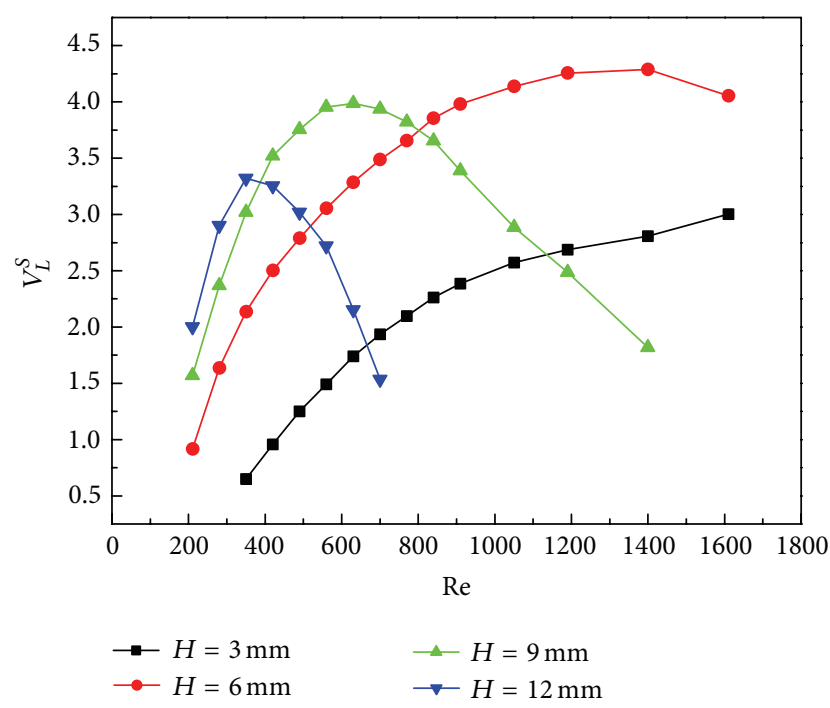

FIGURE 23: Dimensionless secondary vortex length $V_{L}^{S}$ as a function of Re for various $e$ as $h=0.1 \mathrm{~mm}$.

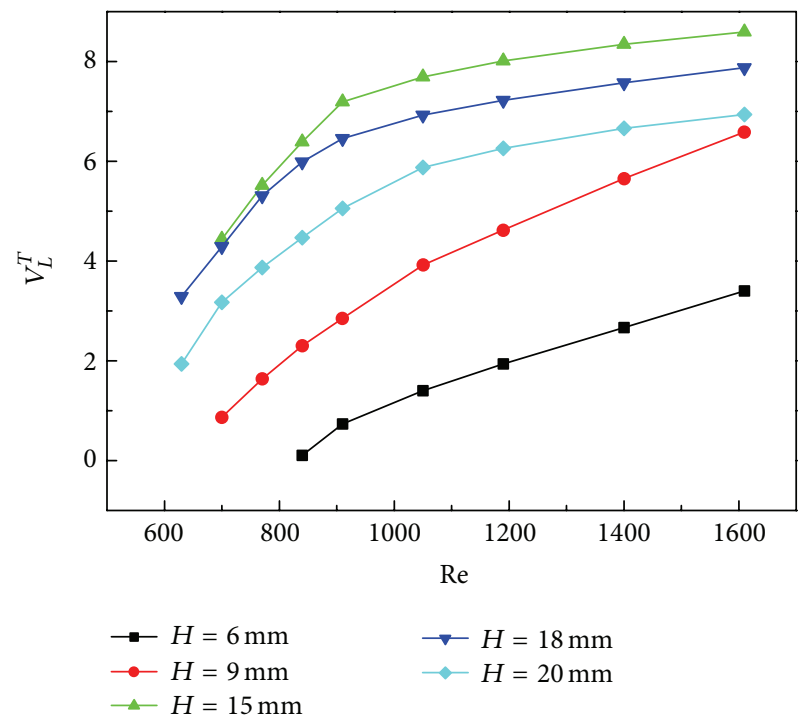

FIgURE 24: Dimensionless tertiary vortex length $V_{L}^{T}$ as a function of Re for various $e$ as $h=0.1 \mathrm{~mm}$.

on the size and amount of vortices, and $h$ has slight effects on the size of vortices. Due to the effect of the sidewall, the relationship between the size of vortices and $h, \mathrm{Re}$, and $e$ has much difference from the relevance in the parallel discs. The lengths of primary, secondary, and tertiary isolated vortices have a linear relationship with $h$. The lengths of the three isolated vortices increase linearly with ascending $e$ as $e$ is small. But when Re and $e$ are large enough, the size of the three vortices decreases linearly.

\section{Nomenclature}

e: Geometrical aspect ratio, $H / R$

$H$ : Distance between two parallel discs $h$ : $\quad$ Distance between seal oil side and upper disc

$\varepsilon: \quad h / H$

$L: \quad$ Length of the nozzle pipe

$R$ : $\quad$ Radius of the nozzle pipe

$r$ : Radial coordinate

$r^{*}: \quad$ Dimensionless radial coordinate, scaled with $R$

$z: \quad$ Axial coordinate

$U$ : $\quad$ Average axial velocity in the nozzle

$P, S, T$ : Primary, secondary, tertiary, and respectively

$S, R: \quad$ Flow separation and reattachment points of a vortex

$V_{L}$ : Dimensionless length of vortex structure, $r_{R}^{*}-r_{S}^{*}$.

\section{Conflict of Interests}

The authors declare that there is no conflict of interests regarding the publication of this paper.

\section{Acknowledgment}

This research is supported by the National Natural Science Foundation of China (Grant nos. 11002007 and 11072011).

\section{References}

[1] D. Sahoo and M. A. R. Sharif, "Numerical modeling of slot-jet impingement cooling of a constant heat flux surface confined by a parallel wall," International Journal of Thermal Sciences, vol. 43, no. 9, pp. 877-887, 2004.

[2] A. S. Goldstein and P. A. DiMilla, "Application of fluid mechanic and kinetic models to characterize mammalian cell detachment in a radial-flow chamber," Biotechnology and Bioengineering, vol. 55, no. 4, pp. 616-629, 1997.

[3] A. S. Goldstein and P. A. DiMilla, "Comparison of converging and diverging radial flow for measuring cell adhesion," AIChE Journal, vol. 44, no. 2, pp. 465-473, 1998.

[4] H. Van Santen, C. R. Kleijn, and H. E. A. Van Den Akker, "Mixed convection in radial flow between horizontal plates-I. Numerical simulations," International Journal of Heat and Mass Transfer, vol. 43, no. 9, pp. 1523-1535, 2000.

[5] H. Van Santen, C. R. Kleijn, and H. E. A. Van Den Akker, "Mixed convection in radial flow between horizontal plates-II. Experiments," International Journal of Heat and Mass Transfer, vol. 43, no. 9, pp. 1537-1546, 2000.

[6] B. B. B. Jensen and A. Friis, "Critical wall shear stress for the EHEDG test method," Chemical Engineering and Processing: Process Intensification, vol. 43, no. 7, pp. 831-840, 2004.

[7] P. S. Moller, "Radial flow without swirl between parallel discs," The Aeronautical Quarterly, vol. 14, pp. 163-186, 1963.

[8] T. Kawaguchi, "Entrance loss for turbulent flow without swirl between parallel discs," Bulletin of JSME, vol. 14, no. 70, pp. 355$363,1971$.

[9] J. C. Hsieh and T. F. Lin, "Effects of jet-to-disk separation distance on the characteristics of mixed convective vortex flow in an impinging air jet confined in a cylindrical chamber," International Journal of Heat and Mass Transfer, vol. 48, no. 3-4, pp. 511-525, 2005. 
[10] F. C. Hsieh, J. H. Wu, J. C. Hsieh, and T. F. Lin, "Unstable vortex flow and new inertia-driven vortex rolls resulting from an air jet impinging onto a confined heated horizontal disk," International Journal of Heat and Mass Transfer, vol. 49, no. 2526, pp. 4697-4711, 2006.

[11] J. G. Detry, C. Deroanne, M. Sindic, and B. B. B. Jensen, "Laminar flow in radial flow cell with small aspect ratios: numerical and experimental study," Chemical Engineering Science, vol. 64, no. 1, pp. 31-42, 2009.

[12] A. Chatterjee, "Multiple vortex formation in steady laminar axisymmetric impinging flow," Computers and Fluids, vol. 37, no. 9, pp. 1061-1076, 2008.

[13] A. Chatterjee, "Newtonian radial entrance flow," AIChE Journal, vol. 46, no. 3, pp. 462-475, 2000.

[14] K. Nakabayashi, T. Ichikawa, and Y. Morinishi, "Size of annular separation bubble around the inlet corner and viscous flow structure between two parallel disks," Experiments in Fluids, vol. 32, no. 4, pp. 425-433, 2002.

[15] G. H. Vatistas, A. Ghila, and G. Zitouni, "Radial inflow between two flat discs," Acta Mechanica, vol. 113, pp. 109-118, 1995.

[16] R. Viskanta, "Heat transfer to impinging isothermal gas and flame jets," Experimental Thermal and Fluid Science, vol. 6, pp. 111-134, 1993.

[17] K. Jambunathan, E. Lai, M. A. Moss, and B. L. Button, "A review of heat transfer data for single circular jet impingement," International Journal of Heat and Fluid Flow, vol. 13, no. 2, pp. 106-115, 1992.

[18] K. P. Angele and B. Muhammad-Klingmann, "PIV measurements in a weakly separating and reattaching turbulent boundary layer," European Journal of Mechanics B: Fluids, vol. 25, no. 2, pp. 204-222, 2006.

[19] Y. Cheng, M. M. Torregrosa, A. Villegas, and F. J. Diez, "Time Resolved Scanning PIV measurements at fine scales in a turbulent jet," International Journal of Heat and Fluid Flow, vol. 32, no. 3, pp. 708-718, 2011.

[20] Y. Liu, Y. Zhao, Y. Cui, and G. Dong, "Numerical simulation and piv study of unsteady flow around hollow cube artificial reef with free water surface," Engineering Applications of Computational Fluid Mechanics, vol. 6, no. 4, pp. 527-540, 2012. 


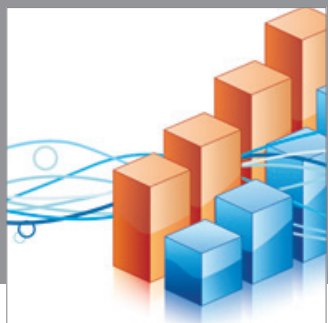

Advances in

Operations Research

mansans

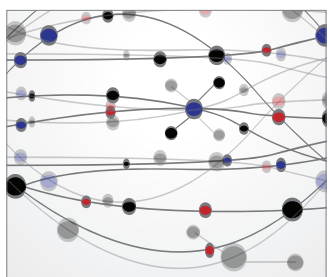

The Scientific World Journal
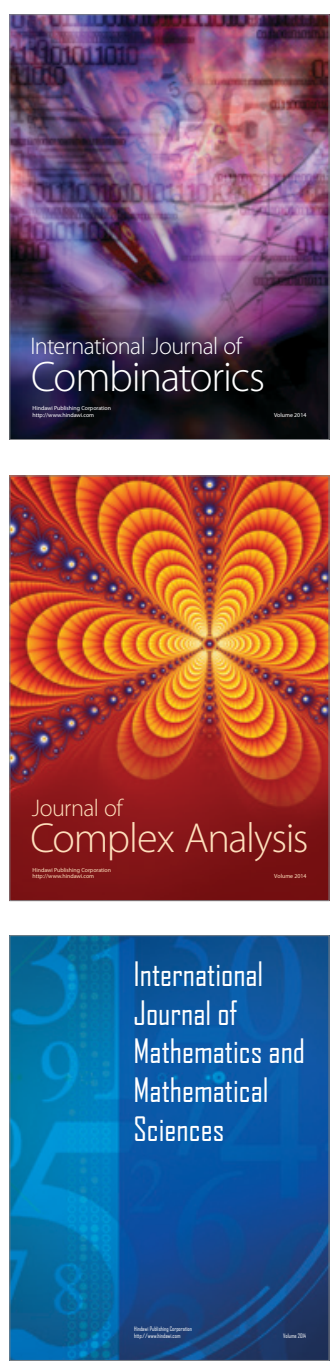
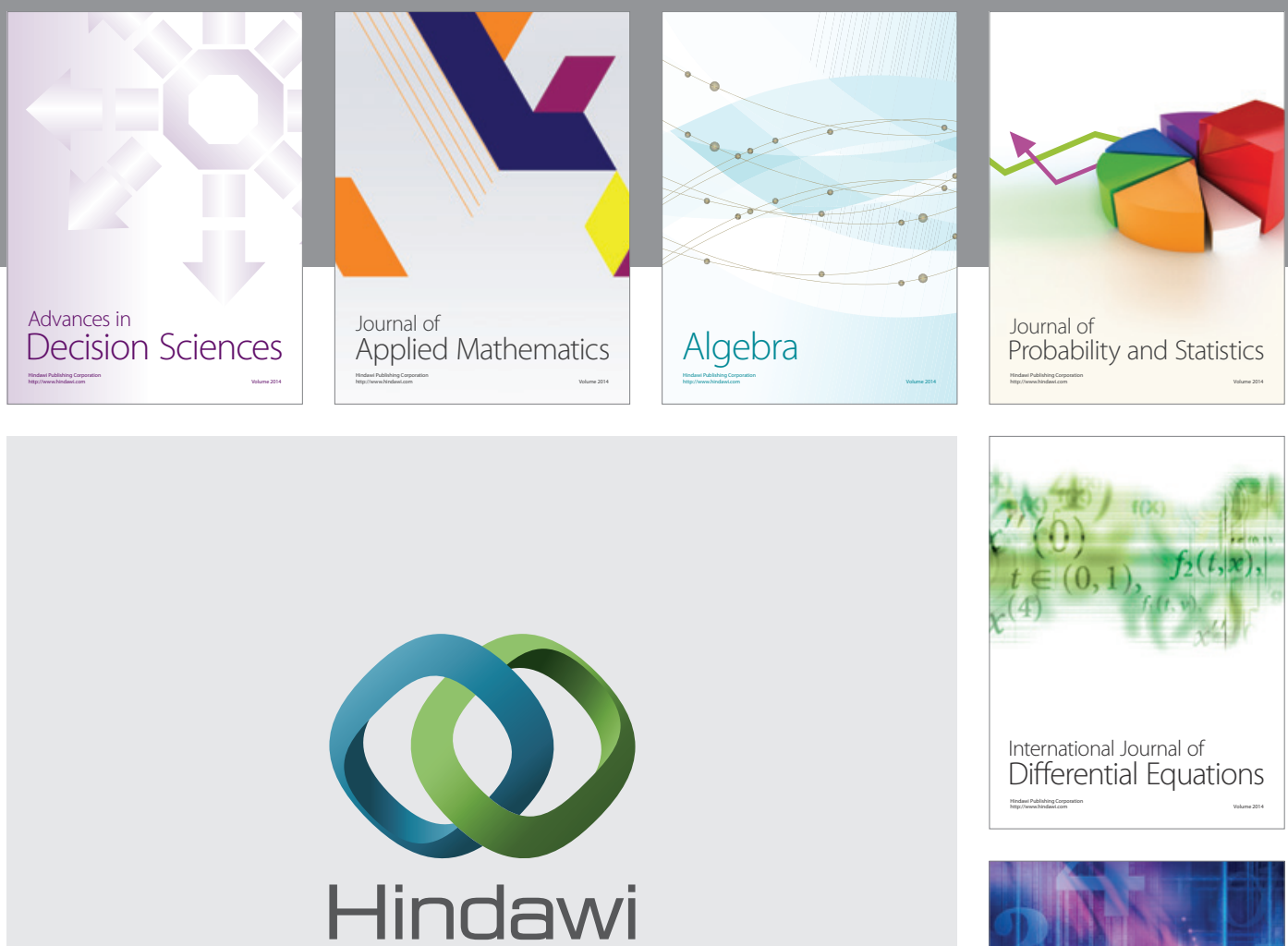

Submit your manuscripts at http://www.hindawi.com
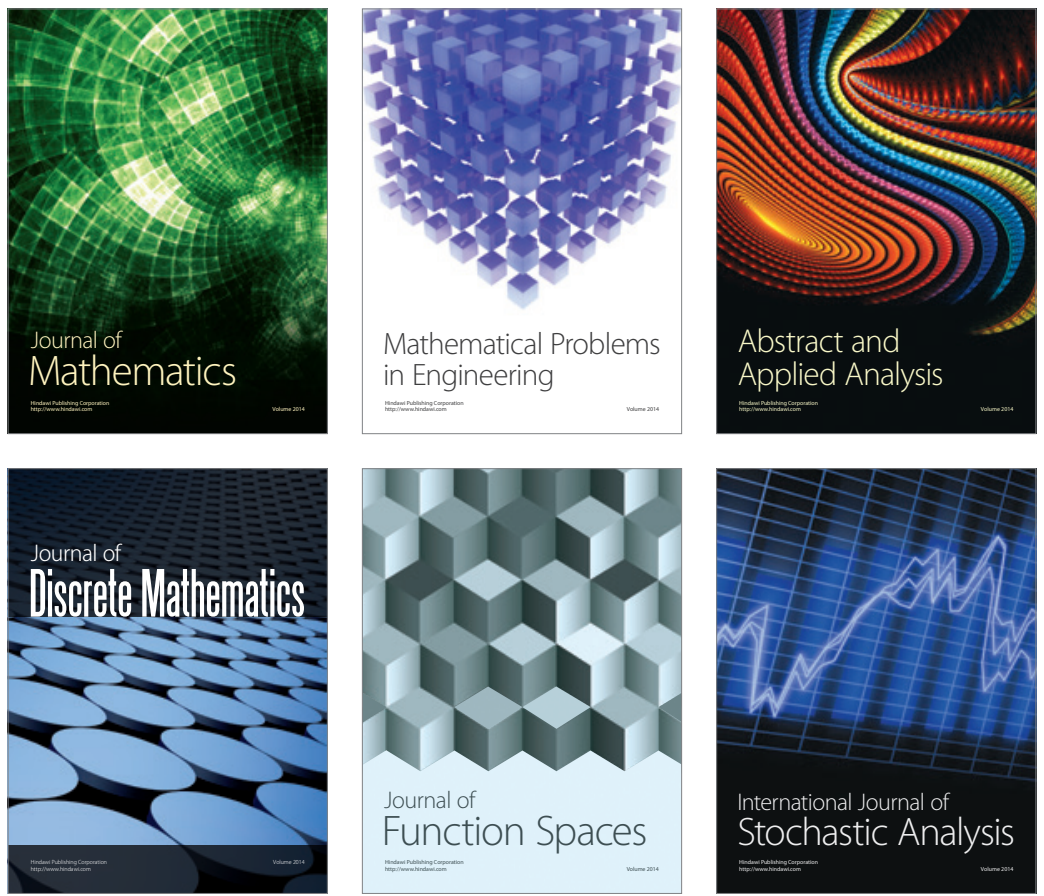

Journal of

Function Spaces

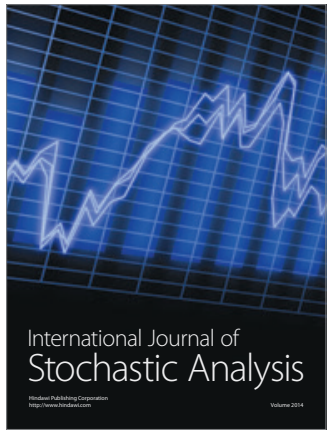

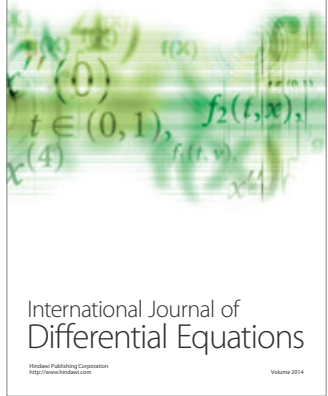
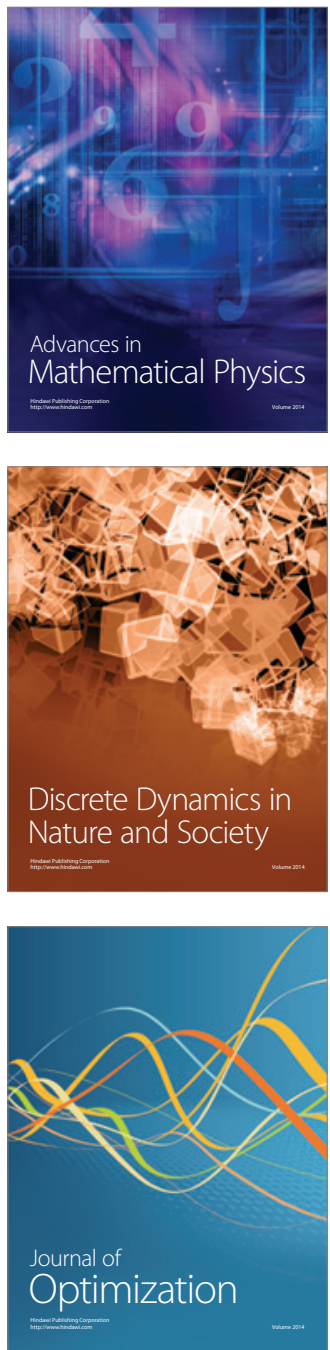